\title{
Dilema da formação matemática em Angola: falta de iniciativas próprias ou de compromisso com a qualidade de ensino?
}

Dilema de la formación matemática en Angola: ¿falta de iniciativas propias o de compromiso con la calidad de enseñanza?

Angolan mathematical training dilemma: lack of own initiatives or commitment to the teaching quality?

\section{Volumen 18, Número 3}

Setiembre-Diciembre

pp. 1-22

\section{Este número se publica el 1 de setiembre de 2018}

DOI: https://doi.org/10.15517/aie.v18i3.33459

\author{
Eurico Wongo Gungula \\ Arnaldo Faustino
}

Revista indizada en REDALYC, $\underline{\text { SCIELO }}$

Revista distribuida en las bases de datos:

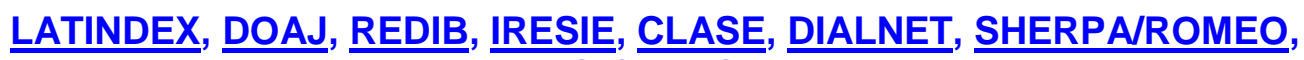
QUALIS-CAPES, MIAR

Revista registrada en los directorios:

ULRICH'S, REDIE, RINACE, OEI, MAESTROTECA, PREAL, CLACSO 


\section{Dilema da formação matemática em Angola: falta de iniciativas próprias ou de compromisso com a qualidade de ensino?}

Dilema de la formación matemática en Angola: ¿falta de iniciativas propias o de compromiso con la calidad de enseñanza?

Angolan mathematical training dilemma: lack of own initiatives or commitment to the teaching quality?

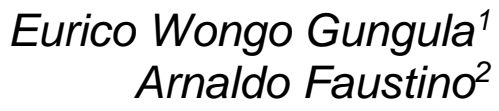

Resumo: Este artigo objetiva abordar o dilema da formação matemática em Angola, através de critérios emitidos por estudantes e professores universitários, devido à insuficiente consciência social sobre a necessidade de melhorar a qualidade de ensino da Matemática, em todos os níveis e subsistemas de ensino. Esta abordagem permitiu identificar aspetos que possibilitam compreender e explicar a trajetória percorrida nesse campo, não só para reestruturar a forma como se inter-relaciona a componente académica, laboral e investigativa, mas também para melhor compreender a realidade matemática angolana, situando-a entre enfoques epistemológicos, metodológicos e tecnológicos que permitem acionar e controlar os elementos, fatores, caraterísticas, contradições e estratégias que contribuam para a transformação qualitativa desse processo. $O$ procedimento metodológico seguido no desenvolvimento desse artigo é misto, pautado, essencialmente, na investigação-ação como um tipo de investigação qualitativa, baseado na observação de fenómenos associados à ação e à resolução de problemas, onde a participação ativa e comprometida do investigador e demais implicados no processo, desempenham um papel fundamental na transformação dessa dinâmica. Os resultados indicam que, apesar de os professores atribuírem, atualmente, maior relevância aos fatores contextuais que dinamizam a construção de conhecimentos matemáticos significativos para os estudantes, estes coincidem com a necessidade de se continuar a elevar os níveis de contextualização dos conteúdos em todos os níveis e subsistemas de ensino, aspeto que contribui para viabilizar o desenvolvimento de uma consciência social e institucional, sobre a pertinência da melhoria da qualidade do ensino da Matemática em Angola.

Palavras chave: ensino de matemática, qualidade da educação, formação de professores, Angola.

Resumen: Este artículo tiene como objetivo abordar el dilema de la formación matemática en Angola, a través de criterios emitidos por estudiantes y profesores universitarios, debido a la insuficiente conciencia social sobre la necesidad de mejorar la calidad de la enseñanza de la Matemática, en todos los niveles y subsistemas educativos. Este enfoque permitió identificar aspectos que posibilitan comprender y explicar la trayectoria recorrida en ese campo, no solo para reestructurar la forma como se interrelaciona el componente académico, laboral e investigativo, sino para comprender mejor la realidad matemática angoleña, lo cual la sitúa entre enfoques epistemológicos, metodológicos y tecnológicos que permiten accionar y controlar los elementos, factores, características, contradicciones y estrategias que contribuyan a la transformación cualitativa de ese proceso, en todos los niveles y subsistemas educativos. El procedimiento metodológico seguido en el desarrollo de ese artículo es mixto, pautado en la investigación-acción, como un tipo de investigación cualitativa basado en la observación de fenómenos asociados a la acción y la resolución de problemas, donde la participación activa y comprometida del investigador y demás implicados en el proceso, juegan un rol fundamental en la transformación de esta dinámica. Los resultados indican que, a pesar de que los profesores asignan actualmente mayor importancia a los factores contextuales que dinamizan la construcción de conocimientos matemáticos significativos para los estudiantes, estos coinciden en la necesidad de seguir elevando los niveles de contextualización de los contenidos en todos los niveles y subsistemas educativos, aspecto que contribuye para viabilizar el desarrollo de una consciencia social e institucional sobre la pertinencia de la mejoría de la calidad de la enseñanza de la Matemática en Angola.

Palabras clave: enseñanza de la matemática, calidad de la educación, formación de profesores, Angola.

1 Rector de la Universidad Óscar Ribas (UÓR), Luanda-Angola. Doctor en Ciencias Pedagógicas, Universidad de Oriente, (UO) y Universidad Máximo Gómez Báez (UNICA), Cuba. Dirección electrónica: euricowongowongo@gmail.com

2 Vice Rector para Asuntos Científicos y de Extensión Universitaria de la Universidad Óscar Ribas (UÓR), Luanda-Angola. Doctor en Ciencias Pedagógicas, Universidad de Oriente, (UO) y Universidad Máximo Gómez Báez (UNICA), Cuba. Dirección electrónica: arnaldo.faustino19@gmail.com

Artículo recibido: 6 de noviembre, 2017

Enviado a corrección: 10 de abril, 2018

Aprobado: 21 de mayo, 2018 


\begin{abstract}
This article aims to address the dilemma of mathematical training in Angola through criteria's issued by university students and teachers, due to insufficient social awareness of the need to improve the quality of mathematics teaching at all educational levels and subsystems. This approach allowed us to identify aspects which made it possible to understand and explain the trajectory in this field, not only to restructure the way it interrelates the academic, technical and investigative components but also to better understand the Angolan mathematical reality, placing it between epistemological, methodological and technological approaches that allows to trigger and control the elements, factors, characteristics, contradictions and strategies that contribute to the qualitative transformation of this process. The methodological procedure followed in the development of this article was mixed and essentially grounded on research action as a type of qualitative research based on the observation of the phenomena associated with action and problem solving, where the active and committed participation of the researcher and others involved in the process plays a key role in transforming this dynamic. The results indicates that although teachers currently attribute greater relevance to contextual factors that stimulate the construction of significant mathematical knowledge for students, they coincide in the need to continually increase the levels of contextualization of contents at all educational levels and subsystems of teaching, an aspect that contributes to the development of a social and institutional awareness about the appropriateness of the improvement of the Mathematics teaching quality in Angola.
\end{abstract}

Key words: mathematics teaching, teaching quality, teacher training, Angola.

\title{
1. Introdução
}

Hoje em dia, a desmotivação dos estudantes pré-universitários e universitários angolanos, no que concerne aos cursos de Licenciatura em Matemática, é muito acentuada e preocupante.

Estes, geralmente, concorrem aos cursos relacionados à Economia, Engenharia Informática, Hidráulica, Agrícola, Electromecânica e Civil, dada a necessidade atual de diversificação da economia, de reconstrução e construção de escolas, hospitais, residências sociais, bancos, aeroportos, pontes, autoestradas, caminhos de ferro, supermercados, entre outras obras sociais.

Não obstante, o limitado enfoque nas potencialidades formativas das matemáticas e como pilar fundamental dos cursos de engenharias, por apenas mencionar uma área do saber, constitui outra barreira que os estudantes enfrentam, por causa da insuficiente orientação profissional sobre as distintas esferas de aplicações praxiológicas.

Para atenuar as limitações mencionadas, é necessário realizar profundas transformações no processo de formação matemática angolano, através da utilização sistemática de procedimentos didático-metodológicos que despertem novos interesses e curiosidades aos estudantes; da implementação de estratégias que desenvolvam formas de pensamento que Ihes possibilite observar e compreender a transcendência da Matemática na solução de problemas sociais.

Nessa perspetiva, torna-se indispensável perceber o valor estratégico da Matemática no processo de desenvolvimento socioeconômico das distintas regiões, visto que, Angola continua nos lugares cimeiros da lista de países que mais contratos de professores com 
formação específica em Matemática realiza, com distintas universidades estrangeiras, para assegurar a continuidade do processo formativo, quer seja em instituições de ensino superior públicas, quer seja em privadas.

Esta situação afeta economicamente o país e revela, nitidamente, a pertinência do aperfeiçoamento do processo de formação de quadros, em geral e, em particular, a formação matemática da nova geração, para que essa possa observar e se esclarecer sobre as potencialidades praxiológicas da Matemática, dado o contexto histórico e social em que vive esta nação africana, nos domínios da educação, economia, saúde pública, reconstrução nacional e da preservação do ambiente.

No que concerne as aplicações da Matemática, encontram-se consideráveis posicionamentos científicos e metodológicos que permitem argumentar e admiti-la como uma ciência com resultados precisos, de procedimentos infalíveis, cujos elementos fundamentais são: operações aritméticas, procedimentos algébricos, definições, teoremas geométricos, assim como posicionamentos que levam a considerá-la como uma ciência acabada, sem espaço para a criatividade e para a inovação, aspeto que merece maior atenção dos professores, em função de criar espaços de intercâmbio de conhecimentos, procedimentos, métodos e técnicas que favoreçam a solução dos distintos problemas reais ou modelados (Da Ponte, 2007; De Guzmán, 2007; Fariñas, 2006; Gungula, Dieguez e Ugartemendía, 2016; Rodríguez, 2013).

Esta perceção da Matemática como ciência acabada, na dinâmica de formação matemática angolana, requer de maior capacidade argumentativa do professor, de modo a reduzir as imprecisões que os estudantes apresentam, sobretudo, na interpretação e resolução de problemas que exigem maiores níveis de abstração, de contextualização e de criatividade (Gungula, Dieguez e Ugartemendía, 2015).

Entre as causas que sustentam essa perceção, destacam-se as seguintes:

1- Insuficiente construção de conhecimentos em forma de atividades que expressem problemas contextuais.

2- Insistente uso de métodos e procedimentos que estimulam, nos estudantes, o desenvolvimento de habilidades memorísticas e reprodutivas.

3- Insistente ênfase em enfoques epistemológicos que não revelam, com suficiente clareza, as potencialidades praxiológicas das matemáticas para estimular o desenvolvimento socioeconômico e tecnológico nacional. 
4- Insuficiente preparação didática, pedagógica e tecnológica dos professores, assim como a descontextualização dos programas de estudo e planos de aulas.

Para contribuir para a transformação qualitativa dessa perceção, torna-se necessário utilizar metodologias ativas, estilos de comunicação matemática que tenham em conta as diferenças individuais e coletivas; assim como, implementar estratégias de construção de conhecimentos que possibilitem visualizar, cada vez mais, a relação existente entre a teoria, a prática e o contexto social.

Os elementos mencionados ganham espaço, sentido e significado no processo de formação matemática angolano, à medida que ajudem aos estudantes, professores e investigadores, a compreender a pertinência de realizar reflexões, críticas e autocríticas, em torno desse processo, para o tornar mais eficiente no futuro, superar os vazios epistémicos, reafirmar os pontos fortes, insistir nos fatores, cujo comportamento demonstrou que são sinérgicos e para não repetir o que uma vez foi fator de debilidade ou desgaste para a melhoria da qualidade de ensino da Matemática.

\section{Problemática da formação matemática em Angola e as perspetivas de desenvolvimento da educação}

A escassez de professores com formação específica em Matemática, para assegurar a continuidade do processo formativo nos cursos de Licenciatura em Matemática, assim como nos cursos, cujos pilares descansam sobre bases matemáticas, constituem, hoje em dia, temáticas de elevada reflexão e debates científico-metodológicos em todos os níveis e subsistemas de ensino em Angola (Faustino, Pérez e Craib, 2016; Gungula, Torrecilla e Puig, 2013; Quitembo, 2010).

Nos Institutos Superiores Pedagógicos e na Faculdade de Ciências da Universidade Agostinho Neto, onde se estuda o curso de Licenciatura em Matemática em Angola, observam-se, atualmente, um reduzido número de estudantes, razão pela que são chamados a implementar estratégias que motivem os estudantes, com ênfase em metodologias ativas, sistemas de procedimentos e recursos tecnológicos orientados a alcançar níveis superiores de contextualização dos conteúdos, de interpretação dos problemas e de aplicação prática dos resultados. 
Não obstante, os professores angolanos com que contam para cumprir esta tarefa, não possuem, desde o ponto de vista didático, pedagógico e tecnológico, uma formação adequada.

Os estilos de comunicação matemática, métodos e meios de ensino-aprendizagem utilizados, não levam suficientemente em conta, a participação ativa dos estudantes na interpretação dos resultados e na aplicação prática destes para a solução de problemas concretos da vida e da profissão (Gungula et al., 2016).

Nesta conformidade, considera-se fundamental, contextualizar os programas de Licenciatura em Matemática ministrados em Angola, ao mesmo nível das licenciaturas ministradas nas distintas universidades estrangeiras, onde o enfoque participativo, tecnológico e interpretativo do estudante na solução de problemas sociais, constituem premissas para avaliar o desempenho profissional do professor.

Desde diferentes fóruns sociais como: conferências didático-metodológicas, eventos científicos nacionais e internacionais, se têm levantado questões em torno dessa situação, visto que os licenciados angolanos selecionados para fortalecer a docência universitária, quer seja em instituições de ensino superior públicas, quer seja em privadas, geralmente limitam-se a dar aulas nos primeiros anos das especialidades onde são colocados, deixando para os professores estrangeiros, as disciplinas mais complexas ou de aplicação prática.

Trata-se de um dilema antigo, que se arrasta desde o ensino básico até ao universitário, devido ao limitado enfoque praxiológico das matemáticas e o papel que desempenham na solução de problemas concretos da vida quotidiana e profissional (Gungula, Faustino e Ugartemendía, 2013).

Por exemplo, quando um sujeito determinado utiliza um telefone, um cartão de crédito, dispositivos eletrónicos para navegar em Internet, calculadoras, computadores, escuta um CD, vê um DVD, conduz um automóvel, apanha um avião, barco, comboio ou um ascensor, está consciente de que, o que faz com que estes artefatos funcionem normalmente são as matemáticas?

Entretanto, se as instituições encarregadas para a formação matemática da jovem geração, orientadas para elevar os níveis de contextualização e de interpretação da produção que realizam, não se comprometerem com a solução da problemática em questão, corre-se o risco de formar professores que, ao assumirem as suas funções, se sintam inseguros para desempenhar com profissionalismo e competência a sua missão (Cuicas, 
Debel, Casadei y Álvarez, 2007; Faustino e Pérez, 2013; Mora, 2005; Moreira e David, 2005; Souza e Garnica, 2004).

Por essa razão, considera-se necessária a superação matemática em áreas específicas, de modo a atenuar a crítica situação que se enfrenta, atualmente, na dinâmica de formação matemática angolana em geral, e, de modo preocupante, no ensino superior (Gungula et al., 2013; Gungula e Faustino, 2013; Faustino, Pérez e Gungula, 2017).

As contradições descritas configuram-se em um círculo de responsabilidades face ao conhecimento matemático que se constrói, à insuficência de professores com formação específica em Matemática e à limitada contextualização das potencialidades da Matemática para estimular o desenvolvimento do pensamento lógico, crítico, autocrítico, reflexivo e criativo dos estudantes.

Nesse âmbito, a sociedade angolana responsabiliza o sistema educativo, pelo fracasso e pela insuficiente visibilidade das aplicações das matemáticas na solução de problemas sociais. Este, responsabiliza o professor pelos baixos níveis de aproveitamento académico dos estudantes.

O professor responsabiliza o estudante pela débil preparação básica em Matemática, quem por sua vez, devolve a responsabilidade ao professor, por causa da excessiva utilização de enfoques epistemológicos e metodológicos que não permitem observar a transcendência dessa ciência na solução de problemas sociais.

Nesse processo de busca do culpado do dilema que se enfrenta no contexto angolano de formação matemática, descarta-se um dos elementos essenciais: a interpretação contextualizada dos resultados da resolução de problemas matemáticos reais ou modelados e, a aplicação prática destes na solução de situações concretas.

As anteriores contradições permitem reafirmar que já é momento de se elaborarem estratégias pedagógicas, metodológicas, educativas ou didáticas próprias, que respondam às necessidades atuais $\mathrm{e}$, debilitem os habituais argumentos usados pela maioria dos professores para justificar o baixo rendimento académico dos estudantes, fundamentando-se na débil preparação matemática que estes revelam, como se as décadas de instabilidade interna não tivessem nenhuma repercussão na supervisão da forma tradicional como os professores davam aulas, assim como, na sua superação contínua, aspeto em que ainda se deve trabalhar com maior compromisso e rigor, para a transformação qualitativa dessa dinâmica. 
É necessário, por outro lado, compreender que independentemente das debilidades identificadas, das limitações analíticas ou interpretativas com que os estudantes ingressam ao ensino superior, é responsabilidade das instituições de ensino superior públicas e privadas atuais, fazer com que os mesmos saiam formados com altos índices de apropriação de conhecimentos, métodos e técnicas, que facilitem a solução de problemas reais ou modelados, para que se desempenhem com competência nas diversas áreas onde forem colocados, como prova do seu compromisso com o desenvolvimento socioeconômico do país.

Segundo autores como Gungula et al. (2013, pp. 251-252); Moreira (2004, p. 4); Quitembo (2010, pp. 22-21); Souza e Garnica (2004, pp. 23-24), bem como as experiências acumuladas pelas universidades brasileiras, portuguesas e cubanas, em situações similares, fundamentam a necessidade de reorientar o processo de formação inicial do professor do ensino básico e as formas de articulação entre conteúdo, pedagogia e prática docente, a partir do papel fundamental da formação específica.

Neste sentido, (Moreira, 2004), recorrendo às contribuições de Lüdke (1994, p. 9), revela que:

(...) já é tempo de se alterar a direção do eixo que vem norteando a licenciatura, fazendo-o centrar-se claramente no lado das áreas específicas. A pesquisa realizada, assim como o conhecimento acumulado pela literatura e a vivência da problemática da área permitem que se afirme este primado. A competência básica de todo e qualquer professor é o domínio do conteúdo específico. Enquanto as unidades específicas não assumirem, como responsabilidade própria, a formação de professores, muito pouco poderão fazer as unidades de educação. Isso não implica, entretanto, que não haja uma importante contribuição da área pedagógica, cuja continuidade deve ser assegurada, mas numa articulação epistemológica diferente com as outras áreas, não numa simples relação temporal de sucessão. Deve-se partir do conteúdo específico, para trabalhar-se a dimensão pedagógica em íntima relação com ele.

Não obstante a estas reflexões, a visão estática como se maneja a problemática de superação matemática em áreas específicas em Angola, quer seja por parte do Ministério da Educação, como das Direções Provinciais de Educação, permitem compreender que as metas traçadas para se elevar a qualidade de ensino, dificilmente alcançarão resultados que visualizem o cumprimento dos objetivos revelados no Plano Nacional de Formação de 
Quadros 2013-2020 (Angola, 2012), se não se contextualizam em função das necessidades de construção de paradigmas que contribuam a dinamizar, cada vez mais, esse processo.

Contudo, os autores desse artigo consideram que, levar adiante o processo de formação matemática com políticas sustentadas em contratos infinitos com profissionais vinculados às universidades européias, americanas e asiáticas, por mencionar apenas um caso, não resolve os verdadeiros problemas relacionados com a dinâmica de formação matemática atual, tão pouco resolverá a do futuro.

É necessário preparar, intencional e estrategicamente, um grupo significativo de investigadores em educação matemática, para que sejam os potenciais dinamizadores de conhecimentos, métodos, procedimentos e técnicas que possibilitem elevar a consciência social sobre o papel que a Matemática desempenha na solução de problemas sociais.

Já é momento de livrar-nos das inacreditáveis pausas pedagógicas que, passivamente, se submetem os estudantes finalistas dos cursos de Licenciatura em Matemática, por escassez de professores angolanos capacitados científica e tecnologicamente para dar continuidade ao processo formativo em pleno século XXI.

Por exemplo, o curso de Licenciatura em Matemática realizado entre 2003 a 2008, no Instituto Superior de Ciências de Educação do Huambo, registou uma série de interrupções, devido à falta de professores angolanos. Entre as disciplinas mais afetadas, destacam-se as seguintes: Análise Numérica (2005/2006), Teoria de Funções (2006/2007), Equações Diferencias e Integrais (2006/2007) e Equações Diferencias com Derivadas Parciais (2007/2008).

Parece enigmático, mas autores como: (Braunschweig e Bethke, 2009, pp. 34-37), coincidem com estas considerações, visto que os resultados das suas investigações revelam que a escassez de professores angolanos qualificados, a falta de escolas em condições, assim como meios de ensino, obrigou as Direções Provinciais de Educação de Luanda, do Huambo, entre outras, a rejeitarem dezenas de milhares de crianças e adolescentes em idade escolar, sobretudo no período compreendido entre 2001 a 2003.

Esta situação despertou a necessidade de problematizar a realidade matemática nacional, de modo a comprometer os futuros Licenciados em Matemática por um lado, e por outro, alertar o Ministério da Educação, sobre a importância da criação de incentivos que contribuam ao fortalecimento do processo de formação matemática, devido as constantes solicitações de professores, fundamentalmente no ensino pré-universitário e universitário. (Gungula et al., 2016). 
Não obstante a estas irregularidades, se reconhece que, apesar da notável valorização que se atribui, atualmente, ao papel que a Didática e Pedagogia podem desempenhar, isso não implica que se deixe de lutar pela criação de espaços de intercâmbio científicos que contribuam a melhorar a dinâmica de formação matemática em Angola, visto que, como ciência básica, constitui a que mais Kwanzas (moeda nacional) extrai anualmente dos cofres do Estado para contratar professores estrangeiros.

Destaca-se ainda que, essa situação agrava-se a cada dia que passa, não só pela insuficiente argumentação do valor científico-metodológico dos conteúdos matemáticos; pela escassez de professores angolanos com formação específia em Matemática, mas pela excessiva inércia do Ministério da Educação e das suas Direções Provinciais, no que respeita à criação de projetos e incentivos que contribuam ao fortalecimento desse processo a partir dos níveis básicos.

Como consequência desse fenómeno, atualmente, não existe nenhuma instituição de ensino superior público ou privado que não tenha, pelo menos, um professor estrangeiro a colaborar no departamento de Matemática ou em unidades equivalentes, incluindo províncias consideradas potencialmente académicas como: Huambo, Huíla, Cabinda, Benguela e Luanda.

Estes aspetos expressam, evidentemente, a gravidade da problemática em questão e motivam a revelar que: apesar da maioria dos estudantes, investigadores, professores préuniversitários e universitários se reservam a criar espaços de reflexões, de intercâmbio científico-metodológico, de críticas e autocríticas encaminhadas ao fortalecimento do processo de formação matemática em Angola, esta forma de pensamento desenvolve-se como base de posicionamentos antigos, antes como estudantes do curso de Licenciatura em Matemática, hoje, como professores e investigadores académicos.

Neste sentido, considera-se que para a transformação qualitativa desse dilema, é necessário desenvolver investigações cujos resultados se traduzam em estratégias para o fortalecimento da dinâmica de formação matemática. Aumentar a quantidade e a qualidade das investigações de cada província, com níveis de contextualização que superem os atuais; valorizar o papel da epistemologia da Matemática para a compreensão das suas raizes, perspetivas e como ferramenta indispensável para estudantes, professores e investigadores. (Gungula et al., 2015).

Não obstante aos aspetos mencionados, considera-se que as contribuições realizadas por autores como: (Gungula, et al., 2013, pp. 491-492), permitem compreender que as 
mudanças que se verificam atualmente na dinâmica de formação matemática angolana, devem partir do contexto de cada região académica, das experiências do passado e das projeções que se traçam para a sua transformação qualitativa.

De outra maneira, não se visualizaria a interpretação das variáveis que incidem sobre o aperfeiçoamento dessa dinâmica, tais como:

1- Programas de estudo desatualizados.

2- Descontextualização dos conteúdos.

3- Acentuada utilização de metodologias e meios de ensino tradicionais.

4- Insuficiente enfoque tecnológico na dinâmica de formação matemática.

5- Insuficiente ênfase na interpretação e aplicação prática dos resultados para a solução de problemas sociais.

6- Insuficiente enfoque na modelação matemática, como arte de expressar, através da utilização de uma linguagem matemática coerente, situações concretas da vida real e solucioná-las, por meio de métodos matemáticos e sistemas computarizados (Software).

7- Insuficiente ênfase na criação de estímulos para estudantes, professores e investigadores.

8- Escassez de professores com níveis de mestrados e doutoramentos em Matemática.

9- Insuficiente preparação didática, pedagógica e tecnológica dos professores.

10- Insuficiente articulação entre a componente académica, laboral e investigativa.

11- Insuficiente estimulação de investigações que resultem em alternativa para a compreensão e difusão das raizes do pensamento matemático angolano.

12- Deficiente articulação do processo formativo da Matemática no ensino superior público e privado.

\section{Bases históricas para a compreensão da problemática da formação matemática em Angola}

Um dos aspetos para a compreensão da problemática da formação matemática em Angola, consiste em seu enfoque histórico, no (des) conhecimento de seus principais expoentes e dinamizadores, visto que, como nação com escassas contribuições internacionais nessa área, em busca de maiores níveis de visibilidade e de interpretação lógica das potencialidades formativas dessa ciência, a investigação das suas bases 
históricas, constitui uma potente ferramenta para a sistematização das raizes do pensamento matemático angolano.

Entretanto, dimensionar o enfoque histórico das matemáticas nesse artigo, não significa resumir as contribuições mais significativas de autores destacados no desenvolvimento da Matemática; implica, porém, descobrir, dentro de seu próprio campo teórico e metodológico, novas inconsistências e solucioná-las, através da utilização de meios didáticos e estratégias que possibilitem visualizar os avanços da ciência e da tecnologia.

Desde esta perspetiva, investigar para à transformação qualitativa dessa dinâmica, não significa preencher os cadernos ou computadores dos estudantes com datas e nomes de autores que contribuíram na difusão da importância das aplicações dos conteúdos matemáticos para o desenvolvimento socioeconômico, para a modernização de distintos países, para a evolução do próprio pensamento matemático, ou enumerar listas de cientistas que se fundamentam na Matemática para melhor abordagem de suas descobertas.

É muito mais do que isso, visto que deve possibilitar, aos estudantes, compreender o elo entre os aspetos enunciados e o contexto em que desenvolvem as suas atividades, quer sejam académicas, laborais ou investigativas, para que desenvolvam formas de pensamento que thes permita resolver problemas reais e modelados, interpretar os resultados em correspondência com as necessidades de aplicação prática, assim como argumentar, lógicamente, o papel que as matemáticas desempenham na evoluçao da ciência e da tecnologia.

Neste sentido, autores como: (Miguel e Miorin, 2004, p. 53) consideram que se pode compreender e permitir que os estudantes percebam a Matemática como uma criação humana; os motivos pelos quais as pessoas fazem matemáticas; as necessidades práticas, sociais, económicas e físicas que servem de estímulo no desenvolvimento de ideias matemáticas; as conexões existentes entre Matemática e Filosofia, Matemática e Religião, Matemática e Lógica, Matemática e pensamento, Matemática e linguagem, Matemática e Música, entre outras.

Para que os estudantes compreendam estes aspetos, é necessário que os professores e investigadores procurem na História da Matemática, elementos que permitam fortalecer a relação entre os conteúdos curriculares, a sua contextualização e as potencialidates de aplicação prática em diversas esferas sociais.

Consequentemente, com as imprecisões que revelam determinados estudantes e professores no que concerne às aplicações práticas das matemáticas na solução de 
problemas concretos da vida quotidiana e profissional, elaboraram-se três questões básicas para melhor compreensão:

1- Para um estudante com limitado esclarecimento das aplicações das matemáticas, quer seja para a solução de problemas do dia a dia, quer seja para a solução de problemas socioeconômicos, que motivação e curiosidade encontra para estudar matemática?

2- Que significado e sentido encontram os estudantes, para se apropriarem com motivação, de conteúdos sem aplicação prática e com elevados níveis de abstração e complexidade?

3- Como fortalecer o estudo de ciências sem antecedentes históricos, do contexto em que os estudantes, professores e investigadores realizam suas actividades académicas, laborais e investigativas?

As anteriores questões ajudam a compreender que a insuficiente visibilidade do elo entre a Matemática escolar, a vida real e o desenvolvimento social, cria uma sombra de dúvidas sobre a importância dessa ciência e de sua aplicabilidade na solução de problemas concretos da vida e da profissão, aspeto que reduz o seu papel no processo de desenvolvimento socioeconômico das nações e no desenvolvimento de habilidades lógicas de pensamento.

Contudo, a escassez de publicações científicas que abordam temáticas relacionadas com as raizes da História da Matemática em Angola, constitui outra barreira que os professores e investigadores em educação matemática enfrentam, quando conseguem criar espaços de intercâmbio que favorecem a motivação dos estudantes no que concerne à transcendência do curso selecionado, quer seja para o desenvolvimento socioeconômico das distintas regiões do país, quer seja para o fortalecimento dos demais cursos préuniversitários e universitários.

Deste modo, encontram-se consideráveis obstáculos para referenciar, contextualmente, os investigadores que, sistemáticamente, contribuem com reflexões, críticas e autocríticas que alertem o Ministério da Educação sobre a necessidade de estimular o desenvolvimento de investigações, cujos resultados permitam compreender e argumentar as raizes do pensamento matemático angolano, como parte da cultura nacional e como estratégia para a melhoria da qualidade de ensino.

Esta situação persiste, porque os insuficientes professores angolanos com formação específica em Matemática, depois da outorga de diplomas de licenciatura, têm mostrado 
insuficiente interesse em continuar com o seu processo formativo e, quando decidem prosseguir, observa-se um desvio para outras ciências, por causa da inexistência de cursos de mestrados e doutoramentos em Matemática nas universidades angolanas.

A inexistência atual dos cursos de pós-graduações mencionados, reduz a consistência científica dos indicadores que se determinam para a compreensão das transformações realizadas em um determinado campo ou objeto de investigação e, dificulta a argumentação praxiológica das propostas que se elaboram, desde uma projeção contextual e universal.

Diante dessa realidade, uma das propostas para o fortalecimento da dinâmica de formação matemática em Angola consiste na sistematização de conhecimentos matemáticos, métodos, técnicas, códigos e símbolos que fundamentam as bases históricas angolanas e, com estas contribuir para a motivação dos estudantes no que concerne ao aprofundamento do estudo dessa ciência.

Desde esse ponto de vista, espera-se que, paulatinamente, os estudantes se motivem e façam parte ativa do processo de enriquecimento da História da Matemática em Angola; contribuam com conhecimentos baseados nas experiências vividas nas suas comunidades e atinjam, por sua vez, domínios similares ou superiores aos que demonstram, quando se abordam aspetos relacionados à História Universal.

\section{Métodos, procedimentos e técnicas}

Buscando bases que confirmem a veracidade da problemática abordada como resultado de uma investigação de natureza mista, utilizou-se o método de investigação-ação, que segundo as contribuições de (Murillo Torrecilla et al., 2010; Thiollent, 1996), é compreendido como um tipo de investigação qualitativa, baseado na observação de fenômenos associados à ação e à resolução de problemas, como alternativa para estimular a cooperação dos demais sujeitos implicados no processo.

Utilizou-se, ainda, o método histórico-lógico, análise documental e a técnica de amostragem aleatória estratificada.

O procedimento utilizado para a elaboração das perguntas realizadas a estudantes e professores universitários, ajusta-se ao estilo Likert e consistiu em atribuir em uma escala de 1 a 5, o espaço correspondente à resposta adequada, de acordo com o nível de conhecimento e de argumentação que possuem sobre a problemática investigada.

Os resultados obtidos foram submetidos à critério de especialistas, onde três professores são da Universidade Católica de Angola-Luanda, três da Universidade Jean 
Piaget de Benguela-Angola, cinco da Universidade Máximo Gómez Báez de Ciego de ÁvilaCuba, cinco do Instituto Superior de Ciências de Educação do Huambo-Angola, um da Universidade Agostinho Neto, Luanda-Angola e, cinco da Universidade Óscar Ribas, Luanda-Angola.

Para a seleção da amostra, aplicou-se a técnica de amostragem aleatória estratificada já utilizada por autores como: (Chile, 2012, p. 10; Lagares e Puerto, 2001, p. 7), definindo-se, por estrato, noventa estudantes dos cursos de Licenciatura em Matemática ministrados nos Institutos Superiores Pedagógicos, assim como a vinte e dois professores de Matemática.

\section{Critérios emitidos por estudantes e professores sobre a problemática da formação matemática em Angola}

Dada a necessidade de fortalecer o processo de formação matemática em Angola, a caraterização do seu estado atual realiza-se por meio de questionários aplicados a estudantes e professores universitários das províncias do Huambo, de Benguela e Luanda.

Selecionou-se aleatoriamente, durante os anos letivos de 2011, 2012, 2013 e princípios de 2016, uma amostra de vinte e dois professores e noventa estudantes universitários, onde 42,2\% (38) destes corresponde ao sexo feminino, e 57,8\% (52) ao sexo masculino, compreendidos entre os 18 e os 35 anos de idade.

Realizaram-se nove perguntas centradas na metodologia utilizada no processo de ensino-aprendizagem da Matemática; no uso de Assistentes Matemáticos na resolução de problemas, comprovação e interpretação dos resultados; na compreensão das causas da desmotivação dos estudantes quanto aos cursos de Licenciatura em Matemática, assim como na relevância da contextualização dos conteúdos e sua aplicação prática na solução de problemas sociais.

$81,8 \%$ dos professores questionados (18), coincidem que a utilização de metodologias ativas no processo de ensino-aprendizagem da Matemática contribui a elevar a participação dos estudantes para o fortalecimento desse processo. Não obstante $90 \%$ dos estudantes (81) exigissem a utilização de metodologias mais ativas, que tenham em conta a participação dos estudantes como centro do processo de ensino-aprendizagem.

$72,7 \%$ dos professores (16) consideram irrelevante a utilização de Assistentes Matemáticos (software) na resolução de problemas matemáticos, na comprovação e na interpretação dos resultados, devido ao limitado domínio e enfoque tecnológico que se atribui ao processo de ensino-aprendizagem da Matemática. Nesse aspeto, 87,8\% dos estudantes 
(79) expressaram que desconhecem a utilidade destas ferramentas, como consequência do excessivo uso de metodologias e meios de ensino tradicionais.

$86,4 \%$ dos professores (19) revelaram que a desmotivação dos estudantes quanto ao estudo dos cursos de Licenciatura em Matemática é devido à débil preparação básica; insuficiente orientação profissional, esclarecimento de suas aplicações, valorização da epistemologia da Matemática, das suas perspetivas e como ferramenta indispensável para estudantes e professores de Matemática.

Expressaram ainda que essa situação tem contribuído a despertar o interesse e a preocupação pelo estudo de processos que facilitem a comunicação matemática, a compreensão, a interpretação, assim como a aplicação prática dessa ciência.

Não obstante, 95,6\% dos estudantes (86) afirmam que a desmotivação em estudar cursos de Licenciatura em Matemática é devido à complexidade desta ciência, aos níveis de abstração necessários para resolver problemas reais ou modelados, ao excessivo tempo dedicado para compreender o enunciado de um exame e transformá-lo em expressões matemáticas que facilitam à sua resolução.

Revelam ainda que, é devido aos reiterativos discursos pré-elaborados por determinados professores em torno do grau de dificuldades que os estudantes que selecionam cursos com elevada carga matemática devem enfrentar, sobretudo, quando estes obtêm classificações negativas nos exames realizados.

$59,1 \%$ dos professores (13) consideram necessário aperfeiçoar o processo de formação matemática através da contextualização dos conteúdos, problemas e resultados.

Não obstante 92,2\% dos estudantes (83) exigissem, por níveis superiores de contextualização dos conteúdos matemáticos, de modo a facilitar sua compreensão, assim como contribuir a desenvolver habilidades analíticas e interpretativas para a solução de problemas reais ou modelados.

\section{Considerações para 0 fortalecimento do processo de formação matemática em Angola}

Uma vez identificados os aspetos que limitam o fortalecimento do processo de formação matemática e analisadas as estratégias elaboradas para o desenvolvimento da educação em Angola, (Lei de Bases do Sistema de Educação, Angola, 2001; Programas curriculares dos cursos de Bacharelato e Licenciatura, Angola, 2007; Plano Nacional de Formação de Quadros 2013-2020, Angola, 2012; Lei de Bases do Sistema de Educação e 
Ensino, Angola, 2016), considera-se que para viabilizar o fortalecimento desse processo, é fundamental ter em conta as seguintes alternativas:

1- Elevar a utilização de metodologias que estimulem a participação ativa dos estudantes, desde os níveis de formação básica, até aos superiores, recorrendo, sistematicamente, à interdisciplinaridade como fator de equilíbrio para a melhoria do processo de ensinoaprendizagem das matemáticas.

2- Que a estratégia de formação de doutores para assegurar a massa crítica mínima das unidades de Investigação, Desenvolvimento e Inovação (IDI), inclua temáticas relacionadas à Matemática, visto que não se expressa no Plano Nacional de Formação de Quadros 2013-2020, (Angola, 2012, pp. 16-32).

3- Que $100 \%$ do corpo docente em educação matemática adquiram uma preparação didática, pedagógica e tecnológica que Ihes permitam um desempenho eficiente no processo de investigação científica, de ensino-aprendizagem, assim como garantir a informação bibliográfica necessária para facilitar, aos estudantes, a apropriação dos conteúdos.

4- Que 100\% do corpo docente estejam capacitados para trabalhar com, pelo menos, um Assistente Matemático (software) na resolução de problemas, na comprovação e interpretação dos resultados, na representação gráfica de funções, entre outras atividades que, no contexto atual, exigem a aplicação destas ferramentas, de modo a captar a atração dos estudantes para futuras investigações que necessitem do uso adequado destes recursos.

5- Que os professores e investigadores em educação matemática aumentem os níveis de contextualização dos conteúdos, por forma a facilitar a visibilidade das aplicações da Matemática, assim como reafirmar a sua trancendência para a solução de problemas sociais.

6- Que se criem, nas distintas regiões académicas do país, espaços de intercâmbio de conhecimentos, métodos, técnicas e estratégias que possibilitem contribuir para a transformação qualitativa do processo de formação matemática.

Embora tivessem ditas em conta as considerações expostas, Teta (2009, p. 34) considera fundamental:

(...) recrutar docentes competentes para as instituições de ensino superior; formar académica e pedagogicamente os docentes recrutados; elaborar programas 
curriculares ao nível das instituições internacionalmente reconhecidas; criar condições para que os professores estejam motivados para os desafíos de desenvolvimento do ensino superior, visto que um docente desmotivado, põe em jogo os avanços futuros de uma sociedade, a ciência, tecnologia e incluso o progresso da humanidade (...)

Com a interpretação das contribuições realizadas por distintos investigadores nacionais e estrangeiros para o fortalecimento do processo de formação matemática, em Angola, resulta indispensável colocar em ação os seguintes postulados, como base para a melhoria da qualidade de ensino:

1- Caráter participativo: Amplia as possibilidades de apropriação de novos conhecimentos, métodos, técnicas e procedimentos, em um clima de total interatividade, humildade científica e respeito pelas diferenças intelectuais.

2- Caráter motivacional: Possibilita elevar a autoestima dos estudantes, professores e investigadores, face à necessidade de resolução de problemas que requerem maiores níveis de contextualização, abstração, argumentação, investigação e de criatividade.

3- Caráter praxiológico: Facilita a visibilidade das distintas aplicações das matemáticas para a solução de problemas concretos, mediante exemplos que tenham um sentido pessoal e social na compreensão dos estudantes, professores e investigadores.

4- Caráter diferenciador: Aumenta as possibilidades para alcançar maiores níveis de generalização de conhecimentos, métodos e técnicas, segundo as necessidades cognitivas de cada estudante, para a solução de um determinado problema.

5- Caráter integrador: Expressa-se nas diferentes formas de ensino, onde seus conteúdos e metodologias estejam em função da necessidade de elevar os níveis de compreensão destes, assim como de interpretação lógica dos problemas e seus resultados.

6- Caráter flexível: Permite a observação das transformações que ocorrem nas estruturas cognitivas dos estudantes, através de seus comportamentos, como expressão de reconhecimento de possíveis erros na seleção de métodos e procedimentos utilizados na solução dos problemas apresentados.

7- Caráter inovador: Permite aumentar, tanto a independência cognitiva dos estudantes, como sua criatividade, mediante o intercâmbio de critérios, de procedimentos de resolução de problemas, assim como de interpretação contextualizada dos resultados. Nos professores contribui ao desenvolvimento de competências profissionais. 
8- Caráter vocacional: Amplia as possibilidades de orientação profissional e a descoberta de novos talentos, através da constante interação dialógica com os estudantes, sobretudo nos últimos anos dos cursos pré-universitários, aspeto que contribui a elevar o número de estudantes, de futuros professores e de investigadores em educação matemática.

\section{Resultados}

Para corroborar a confiabilidade dos resultados e à compreensão da problemática abordada, aplicaram-se as provas não paramétricas de Mann-Whitney e de KolmogorovSmirnov, para duas amostras independentes: grupo de professores e estudantes universitários.

Analisaram-se as respostas de ambos os grupos e os resultados estatísticos indicam que a perceção dos estudantes e professores diverge, significativamente, quanto aos aspetos questionados, (Tabela 1 ).

Tabela 1. Resumo sobre os critérios emitidos por estudantes e professores sobre a problemática da formação matemática em Angola, 2011-2016.

\begin{tabular}{|c|c|c|c|c|}
\hline $\begin{array}{l}\text { Síntese dos } \\
\text { aspetos } \\
\text { questionados. }\end{array}$ & $\begin{array}{l}\text { Utilização de } \\
\text { metodologias } \\
\text { ativas. }\end{array}$ & $\begin{array}{l}\text { Utilização de } \\
\text { Assistentes } \\
\text { Matemáticos } \\
\text { (Software). }\end{array}$ & $\begin{array}{l}\text { Causas da } \\
\text { desmotivação } \\
\text { dos } \\
\text { estudantes. }\end{array}$ & $\begin{array}{l}\text { Pertinência da } \\
\text { contextualização } \\
\text { dos conteúdos. }\end{array}$ \\
\hline \multirow{2}{*}{$\begin{array}{l}\text { Critérios } \\
\text { emitidos } \\
\text { pelos } \\
\text { professores. }\end{array}$} & 18 & 16 & 19 & 13 \\
\hline & $81,8 \%$ & $72,7 \%$ & $86,4 \%$ & $59,1 \%$ \\
\hline \multirow{2}{*}{$\begin{array}{l}\text { Critérios } \\
\text { emitidos } \\
\text { pelos } \\
\text { estudantes. }\end{array}$} & 81 & 79 & 86 & 83 \\
\hline & $90 \%$ & $87,8 \%$ & $95,6 \%$ & $92,2 \%$ \\
\hline
\end{tabular}

Fonte: Elaboração própria dos autores.

Para o processamento dos dados, utilizou-se o Software IBM SPSS Statistics 20, com que se obtiveram as medidas de posição e de dispersão que permitiram resumir a informação.

Os especialistas selecionados coincidem em 97,5\% sobre a necessidade de fortalecer a dinâmica de formação matemática em Angola, de construir estratégias pedagógicas, metodológicas, educativas ou didáticas, que contribuam para atenuar a escassez de professores angolanos com formação específica em Matemática. 
Coincidem, igualmente na pertinência de se elevar os níveis de motivação dos estudantes, quanto ao estudo dos cursos de Licenciatura em Matemática, assim como em realizar ações que visualizem o compromisso pessoal, social e institucional com a melhoria da qualidade do ensino da Matemática, aspetos que permitem afirmar que se cumpriu 0 objetivo da investigação realizada.

\section{Considerações Finais}

Quando se trata de Licenciatura em Matemática em Angola, há que virar a bússola, exclusivamente, para os Institutos Superiores de perfil pedagógico e para a Faculdade de Ciências da Universidade Agostinho Neto, independentemente das suas especificidades.

Não obstante, os professores angolanos com que estas instituições contam para cumprir com sua missão social, revelam, desde o ponto de vista didático, pedagógico e tecnológico, carências que fundamentam a necessidade de se continuar a melhorar a artitulação da componente académica, laboral e investigativa.

Neste sentido, considera-se necessário destacar os seguintes aspetos:

Que 100\% dos projetos de investigação, relacionados com a educação matemática em execução em Angola ou no estrangeiro, obtenham mais de $75 \%$ dos resultados previstos, de modo a responder à excessiva necessidade de professores de Matemática, fundamentalmente, no ensino pré-universitário e universitário.

Que os professores de Matemática estejam, cada vez mais, atentos aos saltos qualitativos produzidos nas estruturas cognitivas dos estudantes, como resultado da utilização de métodos ativos de ensino-aprendizagem e não se limitem a enfocar-se em detalhes de pouca significação para o desenvolvimento de habilidades lógicas de pensamento matemático.

Que se estimulem iniciativas de realização de olimpíadas de Matemática em instituições públicas e privadas, de modo a elevar os níveis de consciência social sobre a necessidade de melhorar a qualidade de ensino dessa ciência.

Que se elaborem estratégias que respondam à necessidade de formação matemática em áreas específicas, que contribuam a fragilizar os tradicionais argumentos usados pela maioria dos professores de Matemática, com vista a justificar os baixos rendimentos académicos dos estudantes, recorrendo sistematicamente, a elementos que expressam a débil preparação matemática apropriada nos níveis precedentes. 
Que se enfatise, cada vez mais, nas aplicações praxiológicas das matemáticas, desde os níveis de formação básica até aos superiores, para que os estudantes desenvolvam formas de pensamento que lhes permita aplicá-los na solução de problemas concretos.

Que os professores compreendam a pertinência de reduzir os níveis de amedrontamento, em torno da complexidade dos cursos de Licenciatura em Matemática, expressos implícita ou explicitamente nos discursos proferidos por estes, aspetos que contribui a elevar a motivação dos estudantes e demonstrar o grau de compromisso pessoal, social e institucional com a melhoria da qualidade de ensino.

Que se publiquem as limitações, debilidades e problemáticas relacionadas à dinâmica de formação matemática em Angola, de modo a contar com critérios de especialistas nacionais e estrangeiros, em torno desse processo, assim como sugestões didáticas, metodológicas e tecnológicas que contribuam para seu aperfeiçoamento.

\section{Agradecimentos}

Os autores agradecem à Direção e aos Especialistas da Revista Actualidades Investigativas en Educación, pela revisão do artigo e decisão tomada para a publicação dos resultados essenciais da investigação realizada.

\section{Referências}

Angola. (7 de outubro, 2016). Lei de Bases do Sistema de Educação e Ensino. Lei número 17/16. Angola: Assembleia Nacional.

Angola. (2012). Plano Nacional de Formação de Quadros 2013 - 2020: programa de acção 2013-2014. Angola: Ministerio do Ensino superior.

Angola, Pró-Reitoria para Reforma Curricular. (2007). Programas curriculares dos cursos de Bacharelato e Licenciatura. Luanda, Angola: Universidade Agostinho Neto.

Angola. (31 de dezembro, 2001). Lei de bases do sistema de educação. Lei número 13/01. Angola: Assembleia Nacional. Recuperado de http://planipolis.iiep.unesco.org/sites/planipolis/files/ressources/angola lei de educaca $\underline{\underline{0 . p d f}}$

Braunschweig, Scott y Bethke, Lynne. (2009). Estudio mundial sobre educación en situaciones de emergencia: informe de Angola [Trad. Daisy Johanna Sánchez]. Revista Magistro, 1(5), 19-47.

Chile, Contraloría General de la República. (2012). Guía práctica para la construcción de muestras. Chile: Unidad Técnica de Control Externo. 
Cuicas Ávila, Marisol, Debel Chourio, Edie, Casadei Carniel, Luisa y Álvarez Vargas, Zulma. (2007). El software matemático como herramienta para el desarrollo de habilidades del pensamiento y mejoramiento del aprendizaje de las matemáticas. Revista Actualidades Investigativas en Educación, 7(2), 1-34. DOI http://dx.doi.org/10.15517/aie.v7i2.9264

Da Ponte, João Pedro. (2007). Investigations and explorations in the mathematics classroom. The International Journal on Mathematics Education, 39(5-6), 419-430.

De Guzmán, Miguel. (2007). Enseñanza de las Ciencias y la Matemática. Revista Iberoamericana de Educación, (043), 19-58.

Fariñas, Gloria León. (2006). Desarrollando del pensamiento complejo. Revista Tiempo de Educar, 7(13), 99-121.

Faustino, Arnaldo y Pérez Luis, Sara. (2013). Utilización de las TIC en la enseñanza de la Estadística en la educación superior angolana. Revista Prisma Social, (11), 0-31.

Faustino, Arnaldo, Pérez Sánchez, Nereyda y Craib Díaz, Elexis. (2016). Habilidades intelectuales en la formación matemática-investigativa angoleña en el perfil Ingenieril. Revista Multiciencias, 16(1), 60-68.

Faustino, Arnaldo, Pérez Sánchez, Nereyda y Gungula, Eurico Wongo. (2017). Formación del Pensamiento Complejo Ingenieril en la Universidad "Óscar Ribas". Telos. Revista de Estudios Interdisciplinarios en Ciencias Sociales, 19(3), 523-544.

Gungula, Eurico Wongo, Dieguez Batista, Raquel, Ugartemendía, Eglys Pérez. (2015). Estrategia didáctica para el perfeccionamiento del proceso de formación interpretativa en la matemática superior. Revista Actualidades Investigativas en Educación, 15(2), 142. DOI: http://dx.doi.org/10.15517/aie.v15i2.18954

Gungula, Eurico Wongo, Dieguez Batista, Raquel y Ugartemendía, Eglys Pérez. (2016). El desarrollo del pensamiento interpretativo del estudiante universitario desde un enfoque didáctico-Matemático. Telos. Revista de Estudios Interdisciplinarios en Ciencias Sociales 18(2), 228-249.

Gungula, Eurico Wongo, Faustino, Arnaldo y Ugartemendía, Eglys Pérez. (2013). El contexto angolano de formación matemática: Un problema que se arrastra desde la base. Revista da Avaliação da Educação Superior, 18(2), 487-499. DOI: http://dx.doi.org/10.1590/S1414-40772013000200013

Gungula, Eurico Wongo e Faustino, Arnaldo. (2013). Actual state of researches in science, technology and society of African universities. Comunicação apresentada em: III international research and practice conference, Westwood, Canada.

Gungula, Eurico Wongo, Torrecilla Díaz, Raudel and Puig Jiménez, Osmany. (2013). Dynamics of mathematical training in Angola and the role of engineering careers. Journal of Education Research and Behavioral Sciences. Apex Journal International, 2(12), 250-253. 
Lagares Barreiro, Paula y Puerto Albandoz, Justo. (2001). Población y muestra. Técnicas de muestreos. España: Management Mathematics for European Schools. Recuperado de http://optimierung.mathematik.unikl.de/mamaeusch/veroeffentlichungen/ver texte/sampling es.pdf

Lüdke, Menga. (1994). Avaliação institucional: Formação de docentes para o ensino fundamental e médio: as licenciaturas. Cadernos CRUB, 1(4), 5-96.

Miguel, Antônio e Miorim, Maria Ângela. (2004). História na educação matemática: propostas e desafios. Brasil, Belo Horizonte: Autêntica.

Mora, David. (2005). Didáctica crítica, educación crítica de las matemáticas y etnomatemáticas. Bolivia: Editorial Campo Iris.

Moreira, Plínio Cavalcanti e David, Maria Manuela Martins Soares. (2005). O conhecimento matemático do professor: formação na licenciatura e prática docente na escola básica. Revista Brasileira de Educação, (28), 50-61. DOI http://dx.doi.org/10.1590/S141324782005000100005

Moreira, Plínio Cavalcanti. (2004). O conhecimento matemático do professor: formação na licenciatura e prática docente na escola básica (Tese para optar pelo grau de Doutor em Educação). Universidade Federal de Minas Gerais, Minas Gerais, Brasil.

Murillo Torrecilla, Francisco Javier, Rodríguez García, Sara, Herráiz Domingo, Noelia, de la Higuera Prieto, Marta, Martínez Solla, Marta, Picazo Zabala, Maribel, Castro Peláez, Irene y Bernal Escámez, Sara. (2010). Investigación Acción. Métodos de investigación en Educación Especial. Recuperado de https://www.uam.es/personal pdi/stmaria/jmurillo/InvestigacionEE/Presentaciones/Curs o 10/Inv accion trabajo.pdf

Quitembo, Alberto Domingos Jacinto. (2010). A formação de professores de Matemática no Instituto Superior de Ciências de Educação em Benguela - Angola. Um estudo sobre o seu desenvolvimento (Tese para optar pelo grau de Doutor em Ciências de Educação). Universidade de Lisboa, Lisboa, Portugal.

Rodríguez, Milagros Elena. (2013). La educación matemática en la con-formación del ciudadano. Telos. Revista de Estudios Interdisciplinarios en Ciencias Sociales, 15(2), 215-230.

Souza, Luzia Aparecida de, Garnica e Antonio Vicente Marafioti. (2004). Formação de professores de matemática: um estudo sobre a influência da formação pedagógica prévia em um curso de licenciatura. Revista Ciência \& Educação, 10(1), 23-39.

Teta, João Sebastião. (2009). Educação superior em Angola. In Marília Costa Morosini (Ed.), Seminário Internacional de Educação Superior da Comunidade de Países de Língua Portuguesa (pp. 30-34) Porto Alegre: EdiPUCRS. Recuperado de http://www.pucrs.br/edipucrs/cplp/educacaosuperior.htm;

http://www.pucrs.br/edipucrs/cplp/arquivos/teta.pdf

Thiollent, Michel. (1996). Metodologia da pesquisa - ação (7ª̣ ed.). Brasil: Editorial Cortez. 